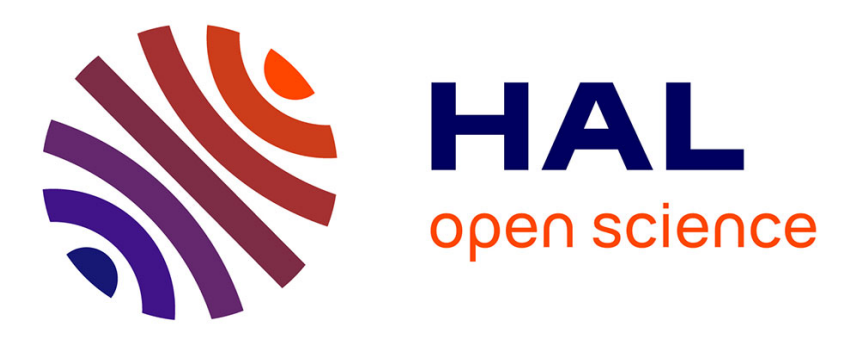

\title{
Evaluation of the noise effects on visible light communications using Manchester and Miller coding
}

Alin Cailean, Barthélemy Cagneau, Luc Chassagne, Valentin Popa, Mihai Dimian

\section{- To cite this version:}

Alin Cailean, Barthélemy Cagneau, Luc Chassagne, Valentin Popa, Mihai Dimian. Evaluation of the noise effects on visible light communications using Manchester and Miller coding. International Conference on Development and Application Systems (DAS),, May 2014, Suceava, Romania. pp.85-89. hal-01207160

\section{HAL Id: hal-01207160 https://hal.science/hal-01207160}

Submitted on 30 Sep 2015

HAL is a multi-disciplinary open access archive for the deposit and dissemination of scientific research documents, whether they are published or not. The documents may come from teaching and research institutions in France or abroad, or from public or private research centers.
L'archive ouverte pluridisciplinaire HAL, est destinée au dépôt et à la diffusion de documents scientifiques de niveau recherche, publiés ou non, émanant des établissements d'enseignement et de recherche français ou étrangers, des laboratoires publics ou privés. 


\section{Evaluation of the noise effects on visible light communications using Manchester and Miller coding}

\author{
Alin-Mihai Cailean ${ }^{1,2}$, Barthélemy Cagneau ${ }^{1}$, Luc \\ Chassagne $^{1}$ \\ ${ }^{1}$ LISV (Laboratoire d’Inginérie des Systèmes de Versailles) \\ University of Versailles Saint-Quentin \\ Vélizy, France \\ alinc@eed.usv.ro
}

\author{
Valentin Popa ${ }^{2}$, Mihai Dimian ${ }^{2}$ \\ ${ }^{2}$ Department of Computers, Electronics and Automation \\ Stefan cel Mare University \\ Suceava, Romania
}

\begin{abstract}
This paper presents the investigation results concerning the negative effects of noise on the data signal in the case of Visible Light Communications (VLC). The motivation of this work was to offer a better understanding of the modifications of the data pulse in the presence of noise. Better understanding of the noise effect on the pulse width can help mitigate it and improve the communication performances. The paper also aims to make a comparative evaluation of two coding techniques used for outdoor VLC: the Manchester code as the code specified by the IEEE 802.15.7 standard in the case of outdoor applications using On-Off-Keying (OOK) modulation and the Miller code, as a possible alternative for Multi Input Multi Output (MIMO) applications. Simulations were performed on messages coded using the two codes for different levels of noise. It seems that in the case of digital processing, the Miller code pulse is less affected by distortions caused by noise. However, in the case of the Manchester code, the higher error tolerance compensates for the pulse distortions. Regarding the Bit Error Ratio (BER), the two codes exhibit similar performances.
\end{abstract}

Keywords- delay modulation; intensity modulation; Manchester coding; noise effect; visible light communication.

\section{Introduction}

Nowadays, the modern society presents a growing demand for wireless communications technologies. This demand is encountered in more and more application fields. Such an application area is the Intelligent Transportation System (ITS). ITS plans to use the wireless data transfer for the so called communications-based safety applications. In these applications, wireless technologies are used to enable Vehicleto-Vehicle (V2V) and Infrastructure-to-Vehicle (I2V) communications with the purpose of increasing the safety and the efficiency of the transportation system. The efforts towards the development of the ITS were confirmed with the publication of the IEEE 802.11p standard [1] for vehicular safety communication. Even if for many years, the radio frequency (RF) based solution were considered the dominating wireless communication technology, in the recent years, VLC came as an alternative emerging technology which could be more appropriate in certain scenarios. Such a scenario is encountered in a high traffic density area, where the increased number of notes that are using the communication medium

This work was supported in part by the University of Versailles SaintQuentin and Valeo Industry. A part of the financial support is granted by the Fond Unique Interminsteriel (FUI) project named Co-Drive, supported by the Pôle de Compétitivité Mov'eo. leads to increased latencies [2, 3], which are unacceptable in traffic safety applications.

The VLC technology takes advantage of the recent efforts made towards the development of the solid state lighting devices [4], such as Light Emitting Diodes (LEDs), which are expected to replace the classical lighting sources in the near future [5, 6]. Besides lighting, LEDs are capable of rapid switching enabling them to add communication to the lighting function. Concerning the VLC receiver, they use light sensing elements which can be photodetector elements or camera systems. Camera systems have the advantage of a wide reception angle. However, the performances of such systems are closely related with the camera's number of frames per second (fps). High speed cameras are able to provide decent BER performances, but due to the high price their usage is limited mostly for laboratory prototypes.

The usage of VLC for transportation related applications was first considered in 1998 [7, 8], and since then much effort has been made on this topic. Now, there are numerous communication systems that use VLC for I2V [9, 10] or for $\mathrm{V} 2 \mathrm{~V}[11,12]$ communication that have been developed and which proved their reliability. Regarding the requirements imposed for the Vehicular Ad-hoc Networks [13], VLC is considered to be able to satisfy them even in real working conditions [14]. VLC is also compatible with platooning as demonstrated in [15]. Even if the communication range of these systems is not as long as for those using RF solutions, multi-hop VLC has proven that by combining short and medium range communications, the overall communication distance can be increased [16]. At this point, VLC usage for transportation related application involves relatively low data rates, from tens of kbps to few Mbps.

The importance of VLC has been certified with the standardization of the wireless optical communications using visible light by the IEEE 802.11.7 standard [17]. The standard comes with three physical layers for different applications and data rates requirements. Within the standard, the PHY I is intended for outdoor, long-range, low data rate applications such as I2V and V2V communication.

This paper presents the simulation results concerning the negative effects of noise on the received data signal, mainly focusing on the pulse width distortions. The analysis is 
performed on messages coded using the Manchester and the Miller code. The simulations were carried based on a signal that was digitally processed. As far as we know, this is the first paper that approaches the issues concerning the noise effect on VLC at the pulse level, investigating the distortion it produces.

\section{The noise influence on the VLC channel}

Intensity Modulation (IM) is considered to be the most appropriate modulation technique for optical communications [18], which is also the case of VLC. In IM, the desired waveform is modulated on the instantaneous power of the carrier. At the receiver side, the data from the modulated light beam is extracted by using Direct Detection (DD). The photodetector produces an electrical current proportional to the incident optical power which is transformed into a voltage by a transimpedance circuit. Next, the signal passes through several filtering and amplification stages until the data signal is reconstructed.

However, the incident power contains not only the data signal but also noise. The outdoor VLC channel is considered to be extremely noisy. Sun light, street light and vehicle lighting systems represent major background noise sources for VLC. These high noise interferences are corroborated with low-level signals due to signal degradation over the distance. The weather conditions, such as fog or rain, also have a negative impact on the VLC data signal. Furthermore, due to the dynamic nature of the traffic, in such applications, the VLC receiver will experience high variations of the SNR. Different mitigation mechanisms such as narrow angle receivers, optical filters, or different signal processing techniques are used at the receiver side, but still, high levels of noise affect the quality of the communication. Therefore, an analysis of the effects of the noisy optical channel on the data signal is strongly required.

The VLC performances are strongly influenced by the communication channel. A VLC channel can be modeled as a baseband linear system, with instantaneous power $X(t)$, output photocurrent $Y(t)$ and impulse response $h(t)$. The channel is subject to signal independent additive noise as shown in Fig. 1 and expressed in eq. 1.

$$
Y(t)=R K(t) \otimes h\left(t^{3}+N_{\text {Tatal }}(t)\right.
$$

where $R$ is the detector responsively (A/W), and $\boldsymbol{Q}$ indicates the convolution.

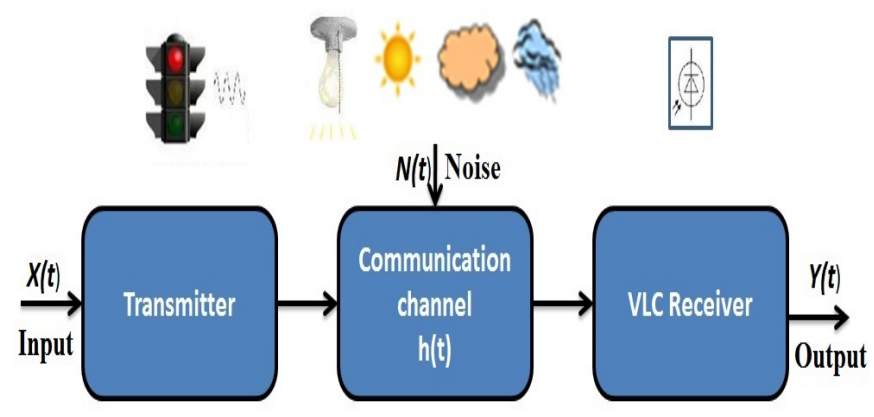

Fig. 1. Simplified VLC channel model.

The noise affecting the VLC channel $\left(N_{\text {total }}\right)$ contains a shot noise component and a thermal noise component, as expressed in eq. 2 :

$$
N_{\text {total }}=\sqrt{N_{\text {sinat }}^{2}+W_{\text {tiserwal }}^{2}}
$$

The shot noise (eq. 3) is proportional to the total optical noise power incident on the receiver. The effect of the shot noise can be minimized by using optical filters, but still this remains a perturbing noise source, limiting the communication's performances. In day-time outdoor communications, shot noise is the dominant noise component.

$$
N_{\text {siba: }}=2 q q I B
$$

where $q$ is the electronic charge ( $q=1.602 \cdot 10^{-19}$ coulombs), $B$ is the detector bandwidth and $I$ is the produced photocurrent.

The thermal noise (eq. 4) is represented by the preamplifier noise, and is the predominant noise source in the absence of background light.

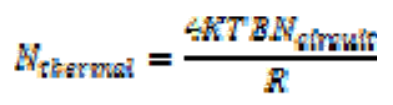

where $K$ is Boltzmann's constant $\left(\mathrm{k}=1.381 \cdot 10^{-23}\right), T$ is the temperature, $N_{\text {circuit }}$ is the circuit noise, and $R$ is the load resistance.

Both the shot noise and the thermal noise are signalindependent and Gaussian. Under these conditions, the total noise affecting the VLC channel can be modeled as signalindependent Gaussian noise.

Regarding the coding techniques, the IEEE 802.15.7 standard for wireless optical communications using visible light defines for the PHY I outdoor applications, the utilization of On-Off-Keying (OOK) with Manchester coding, with data rates from 11.67 to $100 \mathrm{kbps}$. Since the Manchester code is specified by the upper mentioned standard, the noise effect on it is investigated. Even if the Manchester code has numerous advantages, it has the disadvantage of an increased bandwidth requirement. On the other hand, the Miller code [19] clearly outperforms the Manchester code in terms of bandwidth efficiency, making it appropriate for Multiple Input Multiple Output (MIMO) applications as demonstrated in [20]. For this reason, the noise effect on the Miller code is also analyzed. 


\section{Simulation results}

In order to determine the noise influence on the pulse width, several simulations were performed. The simulations involve the coding of random messages using the Manchester and the Miller codes. The messages are transmitted using a modulation frequency of $15 \mathrm{kHz}$ at different SNR levels. The simulated receiver architecture (Fig. 2) assumes the use of a 12 bits ADC module. In order to be able to insure decent signal processing, the sampling frequency is 100 times the data frequency, leading to $1.5 \mathrm{MHz}$. The filtering is performed by two low-pass Butterworth $2^{\text {nd }}$ order cascading filters separated by a partial signal reconstruction block.

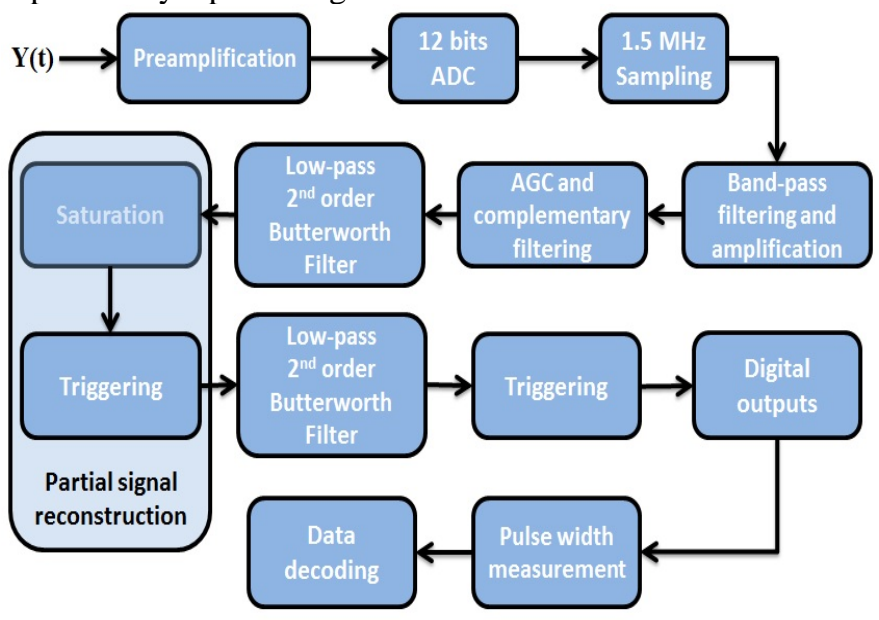

Fig. 2. VLC receiver architecture model.

The usage of the Butterworth filtering [21] was considered due to the fact that it does not introduce ripples to the signal. After filtering, the signal passes through a two level triggering block that outputs the replication of the emitted signal. The pulse widths of the reconstructed messages are measured and analysed in order to determine the noise influence. We mention here that the simulated set up was a simplified one. More complex signal processing techniques, higher order filtering or adaptive threshold algorithms can minimize the effect of the tested noise levels. However, as the SNR continues to depreciate the effects on the pulse widths are similar, meaning that the pulse distortions still occur.

For the purpose of these tests, random messages were coded using Manchester and Miller code. The main parameter of the signal containing the encoded data is the width of the elementary moments of the digital bits. For the Manchester code, there are only two symbols (positive edge and negative edge) and it leads statistically to only two combinations of widths: either one elementary bit width, either twice the elementary bit width. On the other hand, in the case of the Miller code, the memory effect leads to three possible combinations: either one momentary width, or one and a half or twice the width [22]. The possible variations of the pulse widths for the two codes are presented in Fig. 3.

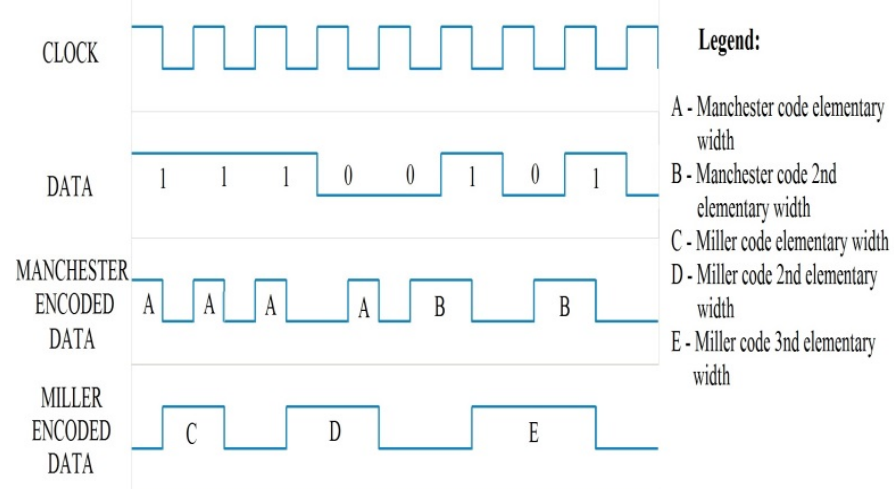

Fig. 3. Illustration of the possible pulse widths in Manchester and in Miller code.

At the receiver side, after passing through the stages mentioned above, the values of $10^{5}$ pulses from each of the pulse widths types were saved and analysed for both codes. It needs to be clarified that for both Manchester and Miller code, a coded frame does not contain an equal numbers of pulses from the two respectively the three pulse widths types. The number of pulses from each category is depending of each message. From this reason the data gathering process continued until the $10^{5}$ number of pulses for each of the pulse widths types was achieved. Even if the purpose of this paper is not message decoding, it is important to mention that decoding is performed based on the identification of the rising and of the falling edges and on the pulse width measurement. In this case it is obvious that pulse distortions above certain tolerances will cause decoding errors.

The results concerning the noise effects on the pulse width are plotted in Fig. 4 for three SNR levels, for the Manchester and for the Miller code. It can be noticed that as the SNR decreases, the pulse widths are more affected by distortion. Also, the number of pulses that are affected is significantly increasing.

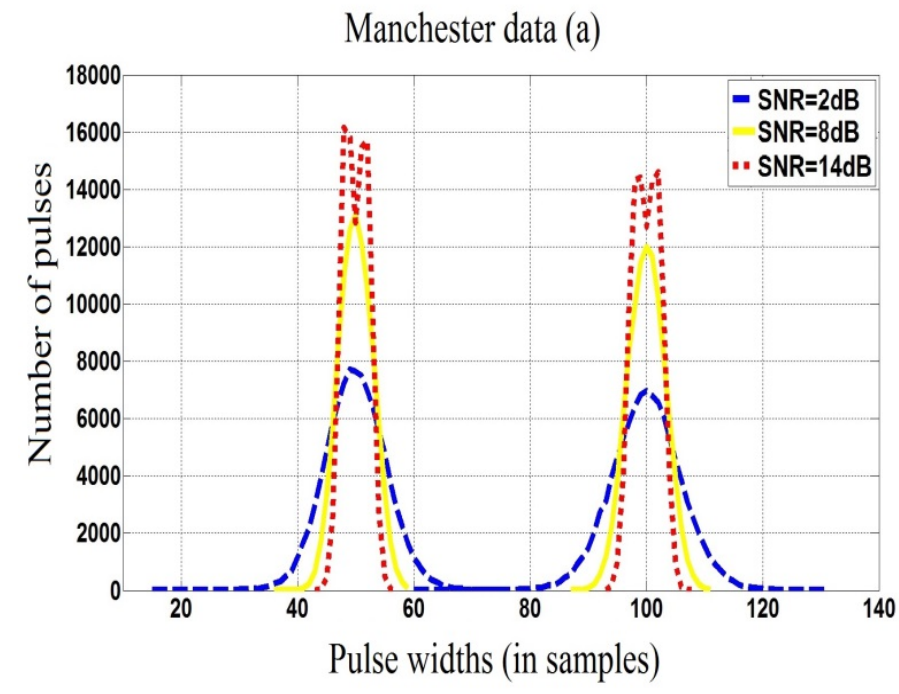




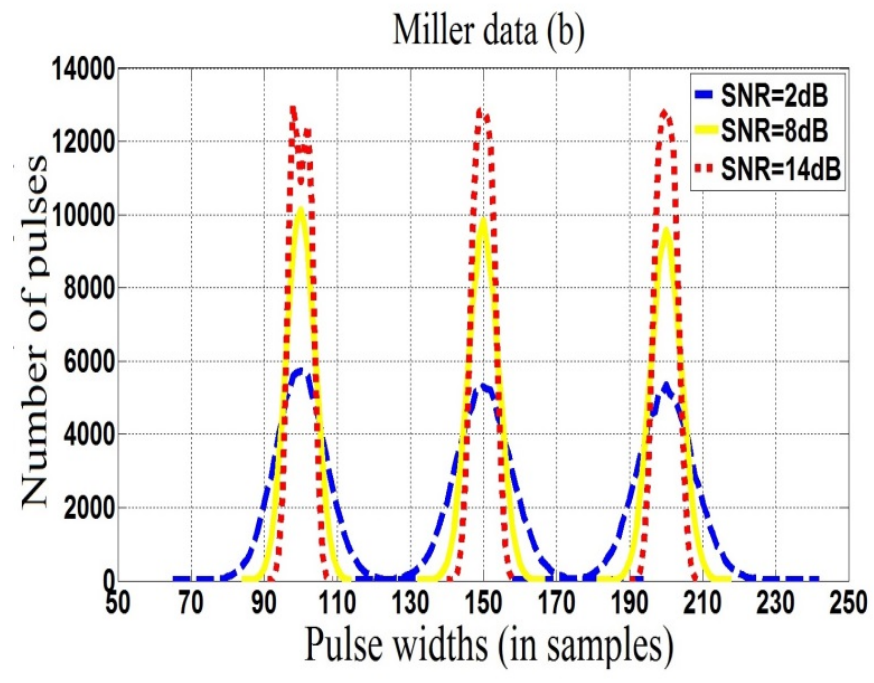

Fig. 4. Histograms of received pulse widths for both Manchester and Miller configurations; Simulation results; a) Manchester case b) Miller case.

It can be observed that in the case of the Miller code, the distortion percentage is not as high as for the Manchester code. This is even more obvious in Fig. 5 where is presented the percentage of pulses that have a pulse width distortion greater than 5\%. The Miller code presents lower distortions because since it has a longer pulse, it uses a greater number of samples per pulse for the filtering.

The pulse width distortions do not represent a significant problem as long as they do not affect the pulse width in a percent that is creating uncertainty for data decoding. Fig. 6 presents the number of pulses that were affected by distortion in a degree that induces decoding errors for the two codes. It can be observed that in the case of the Miller code, the Pulse Error Rate (PER) is significantly higher than the PER of the Manchester code. Due to its nature, the Miller code is more sensitive to variation of pulse length. In its case, pulse width variations of 12.5 to $25 \%$ create uncertainty in decoding. In the case of the Manchester code, the distortion percentage that creates uncertainty is double. However, a Miller pulse has a greater length and therefore it carries more data, making the Bit Error Ratio (BER) similar with the one of the Manchester code.

Another interesting finding revealed during the tests is related to the nature of the errors. In the case of the Manchester code more than $75 \%$ of the errors are due to missing of edges due to insufficient filtering. Basically, due to the noise and because of the limited number of samples per pulse used in the filtering, the signal quality was low and in those cases proper signal reconstruction was unachieved. On the other hand, in the case of the Miller code, the errors are due to the limited tolerances. For example, in the case of a double length Miller pulse, a $12.5 \%$ distortion creates uncertainty in decoding being confused with a middle size pulse.

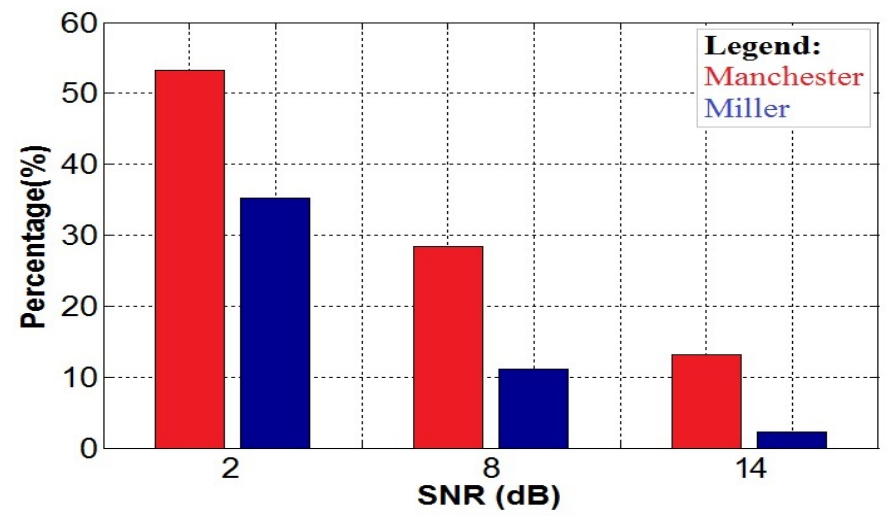

Fig. 5. Percentage of pulses that are affected by a distortion greater than 5\%.

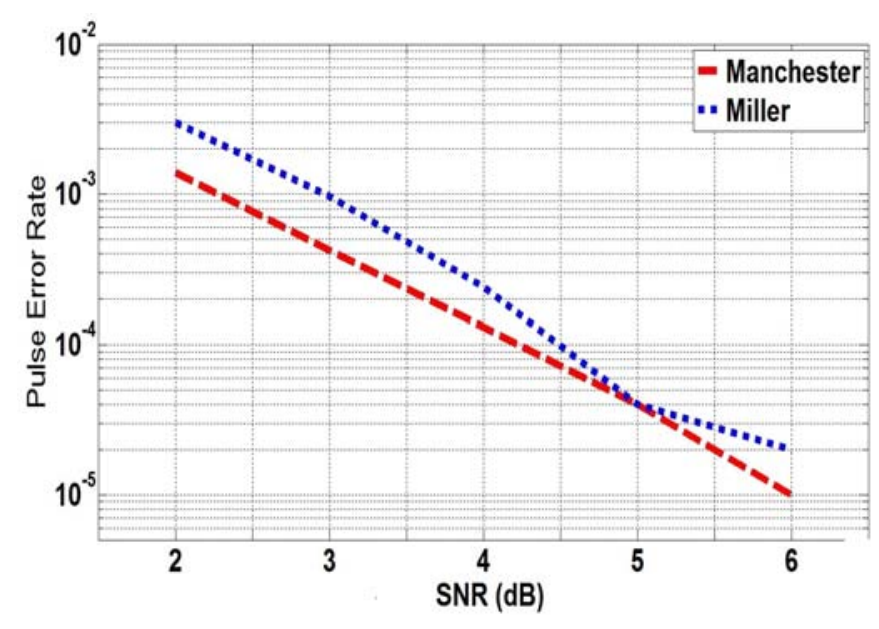

Fig. 6. Pulse error ratio for Manchester and for Miller codes

The purpose of the next tests was to determine the BER performances of the two codes. Short messages of 6 ASCII characters ( $6 * 8$ useful bits) were encoded using the two codes. The structure of the frame also contains several synchronization bits, a start and a stop bit, as presented in Fig. 7.

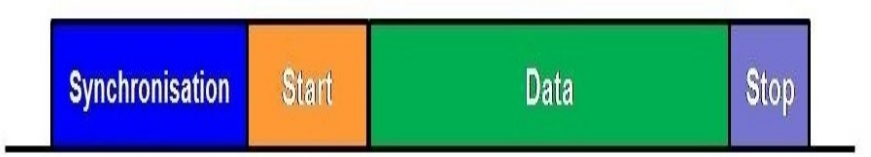

Fig. 7. Structure of the data frame.

At the receiver side, the messages were decoded using the architecture from Fig. 2, for different SNR levels. The BER results for these tests are represented in Fig. 8. It can be observed that at low SNR levels, the Miller code and the Manchester code present almost similar results, with a $10 \%$ better BER in the case of the Miller code. However, as the SNR improves $(4-5 \mathrm{~dB})$, the BER performances seem to get better in the case of the Manchester code, and tend to get even considering a $10^{-7}$ level. 


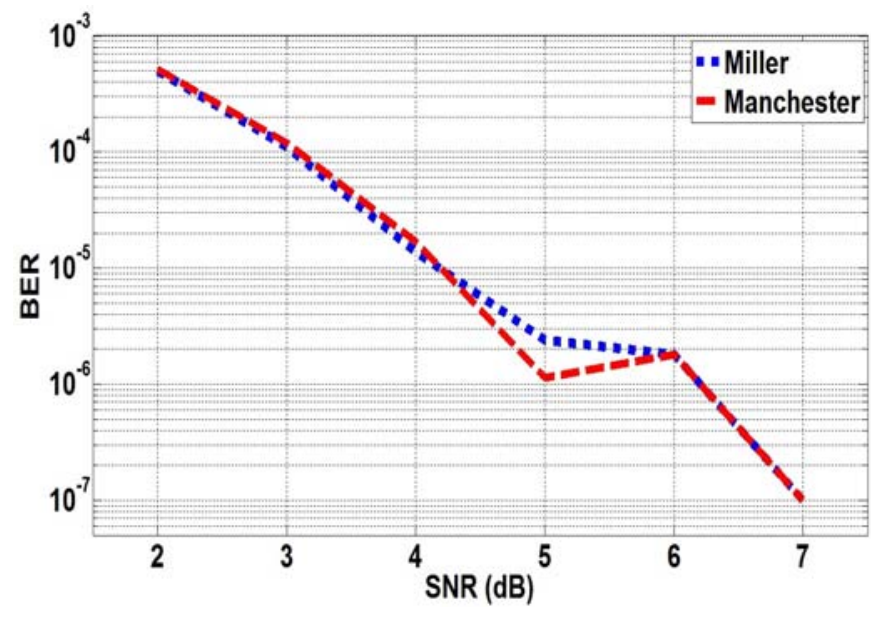

Fig. 8. Bit error ratio for Manchester and for Miller codes

This behavior can once again be attributed to the nature of the two codes. At low SNR levels, the improved filtering performances of the Miller code give it a slight advantage over the Manchester code. As the SNR gets higher, the Manchester coded signal is decently filtered while the Miller coded signal is affected by the stricter tolerance limit. These results can be improved by using higher level filtering, improved pulse reconstruction algorithms or other signal processing techniques, that will mitigate the noise effects on the pulse width to lower SNR levels.

The fact that the Manchester and the Miller code exhibit similar BER performances was also experimentally confirmed in [23] for distances of up to 50 meters.

\section{Conclusions}

This paper presents an analysis over the noise effect on the VLC signal pulse width in the case of digital signal processing. It investigates both the Manchester and Miller code providing simulation results. Within the paper, a VLC receiver architecture is proposed. The results show that as the SNR decreases, the pulse widths are more and more affected by distortion, which causes decoding errors. It can be observed that due to its nature, the Miller coded signal is better filtered, but it is affected by stricter tolerances limit, which is the main cause for errors. In the case of the Manchester code, the digital filtering is less effective but it catches up due to its higher tolerance to pulse width variations. Under these circumstances, the two codes have similar BER results. Since the Manchester and the Miller code have similar performances, but as the Miller code has better channel usage, it can be considered that the Manchester code is adequate for single channel communications whereas in MIMO applications, the Miller code is better suited.

\section{References}

[1] IEEE, "IEEE Std 802.11p, Amendment 6: Wireless Access in Vehicular Environements”, ed: IEEE, July 2010.

[2] Eichler, S., "Performance Evaluation of the IEEE 802.11p WAVE Communication Standard," Vehicular Technology Conference, 2007.
VTC-2007 Fall. 2007 IEEE 66th , vol., no., pp.2199,2203, Sept. 30 2007-Oct. 32007 doi: 10.1109/VETECF.2007.461.

[3] Bilstrup, K.; Uhlemann, E.; Strm, E.; Bilstrup, U., "On the ability of the 802.11p MAC method and STDMA to support real-time vehicle-tovehicle communication," EURASIP Journal on Wireless Communications and Networking 2009, 2009:902414 doi:10.1155/2009/902414.

[4] Branas, C.; Azcondo, F.J.; Alonso, J.M., "Solid-State Lighting: A System Review," Industrial Electronics Magazine, IEEE , vol.7, no.4, pp.6,14, Dec. 2013.

[5] Azevedo, I.L.; Morgan, M.G.; Morgan, F., "The Transition to SolidState Lighting," Proceedings of the IEEE , vol.97, no.3, pp.481,510, March 2009, doi: 10.1109/JPROC.2009.2013058.

[6] Cole, M.; Clayton, H.; Martin, K., "Solid state lighting: The new normal in lighting," PCIC Europe (PCIC EUROPE), 2013 Conference Record, vol., no., pp.1,9, 28-30 May 2013.

[7] Pang, G.K.H., Liu, H., Chan, C.H., Kwan, T., "Vehicle Location and Navigation Systems based on LEDs", Proceedings of fifth World Congress on Intelligent Transport Systems, Paper 3036, Seoul, Korea, 12-16 Oct. 1998.

[8] Pang, G.K.H., Chan, C.H., Liu, H., Kwan, T., "Dual use of LEDs : Signaling and communications in ITS”, Proceedings of fifth World Congress on Intelligent Transport Systems, Paper 3035, Seoul, Korea, 12-16 Oct.1998.

[9] Kasashima, T.; Yamazato, T.; Okada, H.; Fujii, T.; Yendo, T.; Arai, S., "Interpixel interference cancellation method for road-to-vehicle visible light communication," Wireless Vehicular Communications (WiVeC), 2013 IEEE 5th International Symposium on , vol., no., pp.1,5, 2-3 June 2013 doi: 10.1109/wivec.2013.6698237.

[10] Kumar, N.; Lourenco, N.; Terra, D.; Alves, L.N.; Aguiar, Rui L., "Visible light communications in intelligent transportation systems," Intelligent Vehicles Symposium (IV), 2012 IEEE , vol., no., pp.748,753, 3-7 June 2012 doi: 10.1109/IVS.2012.6232282.

[11] Takai, I.; Ito, S.; Yasutomi, K.; Kagawa, K.; Andoh, M.; Kawahito, S., "LED and CMOS Image Sensor Based Optical Wireless Communication System for Automotive Applications," Photonics Journal, IEEE, vol.5, no.5, pp.6801418,6801418, Oct. 2013.

[12] Cailean, A.; Cagneau, B.; Chassagne, L.; Topsu, S.; Alayli, Y.; Blosseville, J-M, "Visible light communications: Application to cooperation between vehicles and road infrastructures," Intelligent Vehicles Symposium (IV), 2012 IEEE , vol., no., pp.1055,1059, 3-7 June 2012.

[13] S. Yousefi, E. Altman, R. El-Azouzi, and M. Fathy, "Analytical Model for Connectivity in Vehicular Ad Hoc Networks", IEEE Transactions on Vehicular Technology, vol. 57, pp. 3341-3356, 2008.

[14] Liu, C.; Sadeghi, B.; Knightly, E. W., "Enabling vehicular visible light communication (V2LC) networks," Proceedings of the Eighth ACM international workshop on Vehicular inter-networking (VANET '11), ACM, New York, NY, USA, 41-50, 2011.

[15] Abualhoul, M.Y.; Marouf, M.; Shagdar, O.; Nashashibi, F., "Platooning control using visible light communications: A feasibility study," Intelligent Transportation Systems - (ITSC), 2013 16th International IEEE Conference on , vol., no., pp.1535,1540, 6-9 Oct. 2013.

[16] Cailean, A.-M.; Cagneau, B.; Chassagne, L.; Topsu, S.; Alayli, Y.; Dimian, M., "Visible light communications cooperative architecture for the intelligent transportation system," Communications and Vehicular Technology in the Benelux (SCVT), 2013 IEEE 20th Symposium on , vol., no., pp.1,5, 21-21 Nov. 2013 doi: 10.1109/SCVT.2013.6736001.

[17] "Ieee draft standard for information technology-telecommunications and information exchange between systems-local and metropolitan area networks-specific requirements-part 15.7: Standard for short-range wireless optical communication using visible light," IEEE P802.15.7/D8, April 2011, pp. 1-306, May 2011.

[18] Kahn, J.M.; Barry, J.R., "Wireless infrared communications," Proceedings of the IEEE, vol.85, no.2, pp.265,298, Feb 1997 doi: 10.1109/5.554222.

[19] Hecht, M.; Guida, A., "Delay modulation," Proceedings of the IEEE , vol.57, no.7, pp.1314,1316, July 1969.

[20] Cailean, A.-M.; Cagneau, B.; Chassagne, L.; Dimian, M.; Popa, V., "Analysis over the Miller code usage in Visible Light Communications under the PHY I layer of the IEEE 802.15.7 standard," Communications 
(COMM), 2014 10th International Conference on , vol., 29-31 May 2014.

[21] Butterworth, S., "On the Theory of Filter Amplifiers," Wireless Engineer, vol. 7, pp. 536,541, 1930.

[22] The mobile communications handbook, Chapter $6,2^{\text {nd }}$ edition, CRC Press LCC, 1999.
[23] Cailean, A.; Cagneau, B.; Chassagne, L.; Topsu, S.; Alayli, Y.; Dimian, M., "A robust system for visible light communication," Wireless Vehicular Communications (WiVeC), 2013 IEEE 5th International Symposium on , vol., no., pp.1,5, 2-3 June 2013 doi: 10.1109/wivec.2013.6698223. 